\title{
OTIMIZAÇÃO USANDO ALGORITMO GENÉTICO DE UM MODELO DE PROPAGAÇÃO BASEADO EM EQUAÇÕES PARABÓLICAS
}

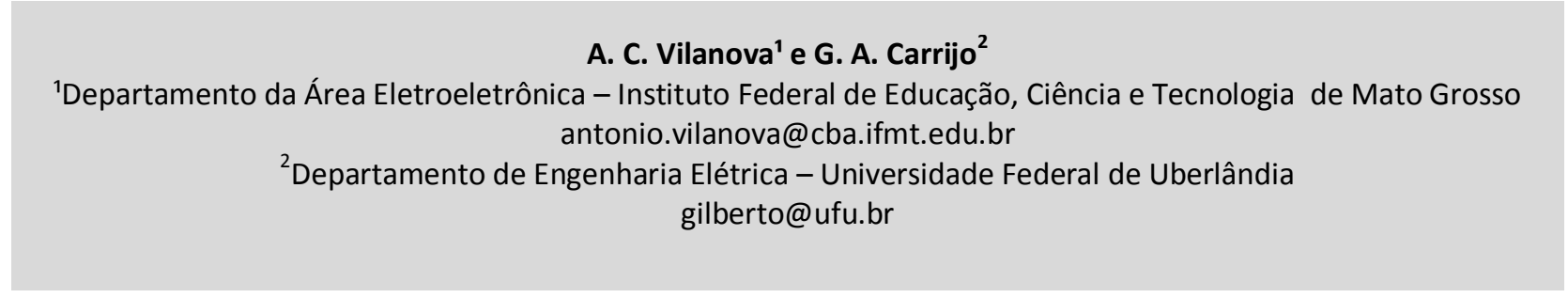

Artigo submetido em agosto/2012 e aceito em dezembro/212

\section{RESUMO}

Este artigo apresenta uma avaliação metodológica para otimizar parâmetros em um conhecido modelo de propagação de ondas de rádio na troposfera. O modelo de propagação é baseado no Divisor de passos de Fourier para resolver equações parabólicas. Nossa abordagem utiliza algoritmo genético para determinar os valores dos parâmetros que maximizem a intensidade de campo em uma determinada posição do observador.
Usando algoritmo genético, o tempo necessário para a busca dos parâmetros ótimos é reduzido significativamente. A avaliação preliminar dos resultados, através da simulação mostra que a nossa abordagem é promissora.

PALAVRAS-CHAVE: Algoritmos Genéticos, Equações Parabólicas, Divisor de passos de Fourier e Custo computacional.

\section{OTIMIZATION USING GENETIC ALGORITHMS OF A PROPAGATION MODEL BASED ON PARABOLIC EQUATIONS}

\begin{abstract}
This paper presents an evaluation methodology to optimize parameters in so called tropospheric radio wave propagation over land model. The propagation model is based on split-step Fourier to solve the parabolic wave equation. Our approach uses genetic algorithm to determine the best parameters toward the
\end{abstract}

maximum field intensity in a given observer's position. By using genetic algorithm the time needed in searching the optimal parameters are greatly reduced. The preliminary evaluation results through simulation show that our approach is promising.

KEY-WORDS: Genetic Algorithm, Equation Parabolic, Split-step Fourier, computational cost. 


\section{OTIMIZAÇÃO USANDO ALGORITMO GENÉTICO DE UM MODELO DE PROPAGAÇÃO BASEADO EM EQUAÇÕES PARABÓLICAS}

\section{INTRODUÇÃO}

Dentre os vários modelos de predição de propagação de ondas eletromagnéticas desenvolvidos nas últimas décadas, como os baseados em Equações Parabólicas (EP), Método dos Momentos (MdM), Diferenças Finitas no Domínio do Tempo (DFDT), Matriz de Linhas de Transmissão (MLT) e Traçado dos Raios (TR), os baseados em Equações Parabólicas têm sido considerados mais atrativos e eficazes e estão sendo amplamente utilizados em modelos de propagação de ondas terrestres por levar em conta a dependência da refratividade com a distância e as irregularidades dos terrenos [1, 2]. Na solução das equações parabólicas, são usadas, principalmente, as técnicas do divisor de passos de Fourier, das diferenças finitas e dos elementos finitos.

O eficiente algoritmo do Divisor de Passos de Fourier para solução de equações parabólicas (EP) utilizado neste trabalho foi desenvolvido em 1973, por Hardin e Tapert [3], e continua sendo muito utilizado nas pesquisas atuais como pode ser visto em $[1,2,4-7]$. Como exemplo de ferramentas desenvolvidas para predição da intensidade do campo elétrico, utilizando a EP com divisor de passos de Fourier, está o pacote de propagação SSPE-GUI, que considera a propagação em um único sentido, desenvolvido em 2005 por [4], e o FSS, que considera a propagação em dois sentidos desenvolvido em 2009, por[5].

Nas ferramentas citadas no parágrafo anterior, o usuário entra com uma combinação de parâmetros como frequência, altura e tilt da antena, largura do feixe, dentre outros, para obter a cobertura de uma determinada região. Cada parâmetro possui uma faixa de valores possíveis como definido em [8]. O conjunto de todas as combinações dos parâmetros dentro dos valores possíveis é denominado espaço de busca.

Encontrar valores ótimos dos parâmetros de interesse em grandes espaços de busca é uma tarefa que exige grande esforço computacional.

Com o objetivo de diminuir esse esforço computacional, os projetos de engenharia requerem além de conhecimentos específicos, em áreas como propagação de ondas, cálculo estrutural, mecânica dos fluídos etc, o uso de técnicas capazes de tratar o grande número de possíveis soluções, trazendo à luz as poucas soluções possíveis, que podem ser chamadas de ótimas. À medida que aumenta a complexidade dos sistemas, torna-se cada vez mais importante o uso da otimização [9].

Os métodos de otimização podem ser divididos em dois grandes grupos: programação linear e programação não linear. A programação linear ( $\mathrm{PL}$ ) tem como objetivo otimizar problemas representados por equações lineares, cujo propósito é minimizar ou maximizar uma função linear, chamada função objetivo. Os métodos de otimização de problemas descritos por equações não lineares utilizam-se da programação não linear (PNL). A PNL divide-se em três grandes 
famílias de métodos: os determinísticos, os enumerativos e os estocásticos. Os métodos determinísticos só podem ser utilizados quando a função objetivo do problema é representada por equação diferenciável e contínua. Os métodos enumerativos, ou seja, por força bruta, verificam todos os pontos possíveis que representem soluções, e tornam-se inviáveis para problemas com grandes espaços de busca. Por outro lado, os métodos estocásticos têm como principal característica a busca pelo ótimo, através de regras de probabilidade, trabalhando de maneira "aleatória orientada". Tais métodos utilizam apenas as informações contidas na função objetivo, não requerendo informações sobre suas derivadas ou possíveis descontinuidades [10].

Problemas que envolvem eletromagnetismo, em geral são complexos, e necessitam de uma abordagem não linear em sua solução. Adicionalmente, não é possível o uso de soluções analíticas nos casos de difícil representação e derivação, sendo necessário o uso de métodos numéricos para contornar esta limitação. Nos casos em que são envolvidas diversas variáveis a serem analisadas é comum o uso de métodos estocásticos na otimização do problema. Este artigo propõe a otimização de parâmetros em um modelo de propagação terrestre baseado em Algoritmos Genéticos, o qual utiliza a técnica de Divisor de Passos de Fourier. Esta abordagem propicia uma significativa redução do custo computacional para a obtenção dos valores ótimos dos parâmetros.

\section{EQUAÇÃO PARABÓLICA}

Neste trabalho, a propagação eletromagnética é resolvida através de Equações Parabólicas e é tratada de forma bidimensional, considerando como variáveis a coordenada $z$, relativa à altura e a coordenada $x$ relativa à distância. Nessas equações, o campo é considerado independente do azimute, que corresponde à coordenada $y$. Esse campo apresenta uma dependência do tempo, $\mathrm{e}^{-\mathrm{j} \omega \mathrm{t}}$, onde $\omega$ é a velocidade angular [11]. Para um meio homogêneo, a componente de campo satisfaz a equação de onda escalar bidimensional de Helmholtz, definida pela equação (1).

$\frac{\partial^{2} \varphi}{\partial x^{2}}+\frac{\partial^{2} \varphi}{\partial z^{2}}+k^{2} n^{2} \varphi=0$,

equação (1)

onde $\varphi=\varphi(x, z)$ é uma das componentes transversais do campo elétrico ou magnético, dependendo da polarização horizontal ou vertical, respectivamente. A variável $k$ é o número de onda e $n$ o índice de refração. A equação (1) aplica-se muito bem em meios com baixo contraste de índice de refração, como os analisados neste trabalho. Para análise de meios com alto contraste e/ou variação abrupta, são necessários formulações semi vetoriais ou vetoriais [12]. Para propagação em ângulos próximos ao eixo $x$, direção paraxial, utiliza-se a equação reduzida (2).

$$
\varphi(x, z)=e^{-j k z} u(x, z),
$$

equação (2) 
pois a variação lenta de $x$ lhe confere convenientes propriedades numéricas. A equação parabólica padrão em termos de $u$ é dada pela equação (2).

$\frac{\partial^{2} u}{\partial z^{2}}+2 j k \frac{\partial u}{\partial x}+k^{2}\left(n^{2}-1\right) u=0$.

equação (3)

No vácuo como $n$ não varia $\operatorname{com} x$ e $z$, a equação parabólica padrão é descrita pela equação (4):

$\frac{\partial^{2} u}{\partial z^{2}}(x, z)+2 j k \frac{\partial u}{\partial x}(x, z)=0$.

equação (4)

Com o objetivo de resolver a equação (4) no domínio espectral que é mais simples e retornar para o domínio espacial, utiliza-se a transformada de Fourier e sua inversa, respectivamente.

Aplicando a propriedade da transformada de Fourier $\mathrm{F}(\partial \mathrm{u} / \partial \mathrm{x})=\partial(\mathrm{Fu}) / \partial \mathrm{x}$ na equação (4) resulta na equação (5).

$-4 \pi^{2} \rho^{2} U(x, \rho)+2 j k \frac{\partial U}{\partial x}(x, \rho)=0$

Trata-se de uma equação diferencial ordinária e pode ser resolvida de forma fechada, resultando na equação (6).

$U(x, \rho)=e^{-\frac{2 j \pi^{2} \rho^{2} x}{k}} U(0, \rho)$

equação (6)

onde:

$\rho=\mathrm{k} \cdot \operatorname{sen}(\theta) \quad$ É a variável no domínio espectral

k

É o número de onda

$\theta$

É o ângulo de propagação com a horizontal.

$U(0, \rho)$

É o campo na origem, no domínio espectral, obtido pela transformada$$
U(x, \rho)
$$
de Fourier do campo na origem, no domínio espacial, na uma altura z.

É o campo a uma distância $x$ da origem, no domínio espectral, na

O campo no domínio espacial na altura $z$, a uma distância $\mathrm{x}$ da origem, é obtido pela transformada inversa da equação (6),

$u(x, z)=F^{-1}\left\{F\left\{e^{-\frac{2 j \pi^{2} \rho^{2} x}{k}} U(0, z)\right\}\right\}$

equação (7)

Onde: 
$u(0, z) \quad$ É o campo na origem, na altura z e no domínio espacial, obtido diretamente do diagrama de radiação da antena.

$u(x, z) \quad$ É o campo a uma distância $\mathrm{x}$ da origem, na altura $z$, e no domínio espacial.

Analisando a equação (7), verifica-se que foi utilizado o par de transformada de Fourier. Primeiro utilizou-se a transformada para converter o campo, na origem, do domínio espacial para o domínio espectral, facilitando a solução da equação (5) que é uma equação diferencial ordinária. Uma vez obtida a solução para o campo, a uma distância $x$ da origem, no domínio espectral, pela equação (6), utilizou-se a transformada inversa de Fourier para se converter o campo, a uma distância $\mathrm{x}$ da origem, novamente para o domínio espacial.

Para o campo a uma distância $x$ da origem, no domínio espectral, $\boldsymbol{U}(\boldsymbol{x}, \boldsymbol{\rho})$, é utilizada a teoria da imagem para garantir a condição de contorno de campo zero na superfície,

$\varphi(0, z)=A\left(z-z_{0}\right)-A^{*}\left(z+z_{0}\right)$.

equação (8)

No domínio espectral, o campo na origem é expresso pela equação (9).

$U(0, \rho)=f(\rho) e^{-j \rho z_{0}}-f^{*}(\rho)^{j \rho z_{0}}$.

equação (9)

onde $\mathrm{f}(\rho)$ é o diagrama de radiação e $\mathrm{z}_{0}$ a altura da antena. A altura da antena e a frequência são os parâmetros a serem otimizados pelo Algoritmo Genético. Neste trabalho foi utilizado o diagrama de radiação Gaussiano definido pela equação (10).

$$
f(\rho)=e^{\left[\frac{-\rho^{2} 2 \ln 2}{4 k \operatorname{sen}\left(\theta_{B W} / 2\right.}\right]}
$$

onde $\theta_{\mathrm{BW}}$ é a largura de feixe.

A inclinação da antena, tilt, pode ser alterada de um ângulo $\theta_{\text {elev }}$ substituindo $\boldsymbol{f}(\boldsymbol{\rho})$ por

$$
f\left(\rho-k \operatorname{sen}\left(\theta_{e l e v}\right)\right) \text {. }
$$

O campo distante forma um par de transformada de Fourier com o diagrama de radiação da antena.

O método de Equações parabólicas converte o problema de propagação na troposfera em um problema de um valor inicial que pode ser resolvido, empregando-se métodos numéricos, marchando com a distância em conjunto com as condições de contorno apropriadas.

O algoritmo Divisor de Passos de Fourier parte da origem e avança, com passos $\Delta \mathrm{x}$, até atingir o alcance máximo desejado. 
O campo para a distância $\mathrm{x}+\Delta \mathrm{x}$ é calculado de forma aproximada pela equação (12).

$$
u(x+\Delta x, z)=e^{j k\left(n^{2}-1\right) \Delta x / 2} F^{-1}\left\{e^{-j p^{2} \Delta x / 2 k} F\{u(x, z)\}\right\}
$$

\section{ALGORITMOS GENÉTICOS}

O Algoritmo Genético (AG) é um algoritmo de otimização estocástico baseado na teoria de Darwin de seleção natural e reprodução genética [13]. Este princípio de seleção privilegia os indivíduos mais aptos com maior longevidade e, portanto, com maior probabilidade de reprodução.

No algoritmo genético, é necessária a avaliação da adaptabilidade de cada indivíduo ao meio. $\mathrm{O}$ meio significa o problema e a adaptabilidade do indivíduo a esse meio é medida por um valor obtido através das características desse indivíduo para o problema em questão, quantificadas por uma função aptidão. $O$ valor da aptidão indica o quanto a solução representada pelo indivíduo é satisfatória para o problema[14].

O AG apresenta uma sequência de execução de tarefas que envolvem:

- Definição da função objetivo do problema e dos parâmetros a ser otimizados;

- Codificação dos parâmetros a ser otimizados como genes;

- Criação de uma sequência de genes para formar um cromossomo;

- Gerar uma população inicial de possíveis soluções;

- Avaliar e atribuir valores à aptidão de todos os indivíduos da população;

- Realizar a reprodução através dos operadores seleção, cruzamento e mutação para produzir os indivíduos da próxima geração.

Uma população inicial de soluções é gerada aleatoriamente. Em seguida, os indivíduos da população são avaliados e é verificado se a solução desejada foi encontrada. Se a solução não foi encontrada, os indivíduos são submetidos aos operadores genéticos para formar os indivíduos da próxima geração. $O$ processo se repete até que um critério de parada seja atingido. Os principais critérios de parada são: o número máximo de gerações, erro máximo admissível e diversidade genética.

A aptidão dos indivíduos evolui de acordo com os operadores genéticos de modo que há uma tendência de que, na média, os indivíduos tornem-se cada vez mais aptos à medida que o número de gerações aumenta. 
Para avaliar a evolução dos indivíduos, há algumas medidas de desempenho. A principal é a curva de desempenho que compara a evolução dos valores médio e máximo da população em cada geração. Outras medidas como fitness mínimo e desempenho on-line podem ser vistas em [14].

Com a curva de desempenho representada graficamente, é possível uma análise do que ocorreu durante a execução do algoritmo genético. Quando o valor médio aproxima-se do valor máximo indica que o algoritmo convergiu.

\section{O APLICATIVO EP-AG}

O aplicativo Equações Parabólicas otimizado por Algoritmo Genético (EP-AG), apresentado neste trabalho, é constituído de duas partes principais [4]. Uma relativa ao modelo de propagação; e a outra ao mecanismo de otimização. A interface gráfica possui campos para entrada de parâmetros, visualização dos resultados, monitoramento do processo e uma área para apresentação de gráficos. Os parâmetros do AG inseridos pela interface são número de gerações, tamanho da população e taxas de cruzamento e mutação, enquanto os principais parâmetros inseridos para o modelo de propagação são largura de feixe e tilt da antena, altura máxima e alcance máximo do terreno, limites inferior e superior da frequência e da altura da antena, nome e caminho do arquivo com o perfil do terreno. Na interface, é possível acompanhar a evolução do processamento em que apresenta o número da geração atual e, para cada indivíduo gerado, os valores da frequência, a altura da antena e a aptidão. Também são visualizadas as aptidões máxima e média da geração inicial e da última geração processada.

\section{METODOLOGIA}

A Equação Parabólica é resolvida pelo Divisor de passos de Fourier. O algoritmo do Divisor de passos de Fourier parte da origem e avança com passos $\Delta \mathrm{x}$ até atingir o alcance máximo desejado. Para cada passo, o campo é determinado ao longo da direção vertical e o perfil do campo é armazenado. A representação dos perfis verticais está ilustrada na Figura 1.

O campo para a distância $\mathrm{x}+\Delta \mathrm{x}$ é calculado aproximadamente pela equação (12).

O aplicativo EP-AG permite configurar o número de perfis verticais e número de pontos de observação por perfil. Neste trabalho, O EP-AG foi configurado para mil perfis verticais, com 256 pontos de observação cada. Portanto, após cada execução do aplicativo, é gerada uma matriz 1000 x 256 que armazena os valores de campo de todos os pontos de observação. $O$ aplicativo permite traçar o contorno do campo para a área do terreno analisado, convertendo os valores do campo de todos os pontos de observação em cores ou escala de cinza. Também é possível traçar o perfil vertical do campo em uma determinada distância. 
Para obter os resultados apresentados neste trabalho, o aplicativo EP-AG foi executado com três diferentes configurações de terreno.

Na primeira configuração, mostrada na Figura 2, o terreno tem alcance de $20 \mathrm{~km}$, altura de 300 $\mathrm{m}$, e um obstáculo gume de faca de $150 \mathrm{~m}$ a $8 \mathrm{~km}$ da antena transmissora que está localizada a uma altura de $150 \mathrm{~m}$ e o transmissor opera na frequência de $100 \mathrm{MHz}$, com largura de feixe de $1^{\circ}$. Esta configuração é utilizada para analisar a influência do ângulo tilt.

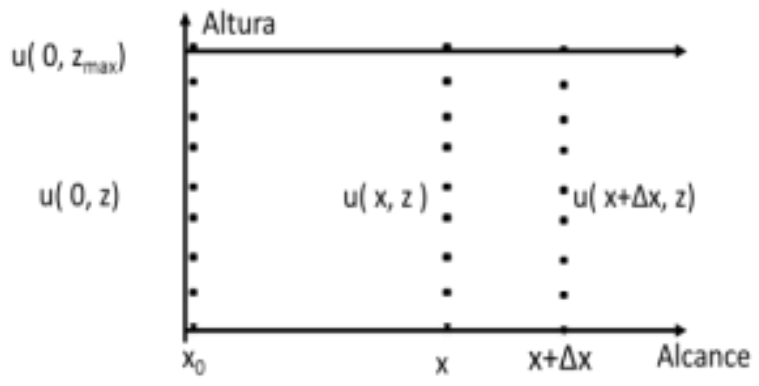

Fig.1 Representação dos Perfis verticais do campo.

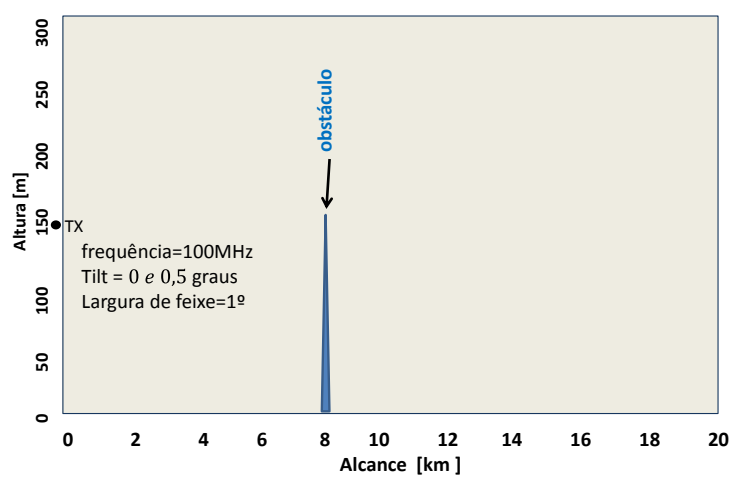

Fig. 2 Configuração para análise da influência do ângulo tilt.

Nas outras duas configurações mostradas na Figura 3, o perfil do terreno tem altura máxima de $250 \mathrm{~m}$ e alcance máximo de $140 \mathrm{~km}$. A largura de feixe fixada em $1^{\circ}$ e o tilt em $0^{\circ}$. Nestas configurações, estão destacados três observadores denominados observador 1, observador 2 e observador 3, distantes da antena 20, 70 e $110 \mathrm{~km}$, respectivamente, e com altura de 50, 80 e 50 metros respectivamente. Estas configurações foram utilizadas para a análise da influência dos obstáculos gume de faca e dos perfis de refratividade.

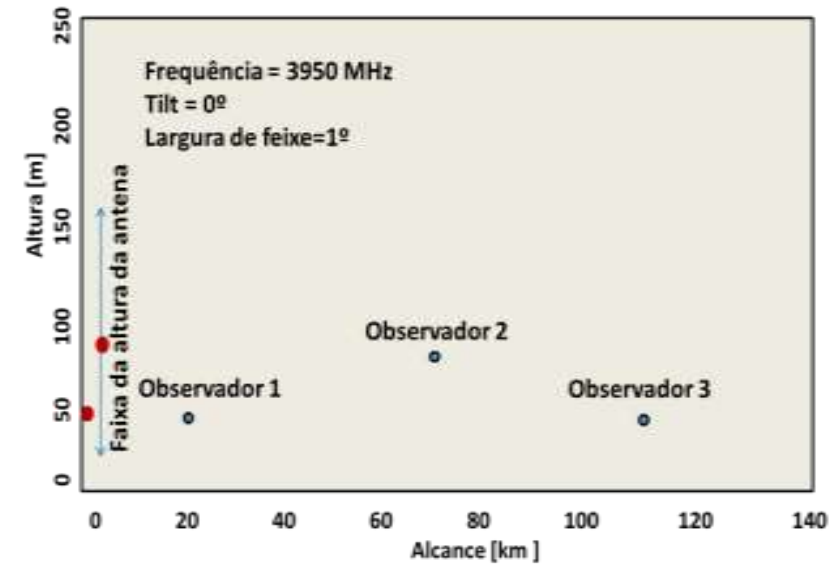

3.a) Configuração sem obstáculos.

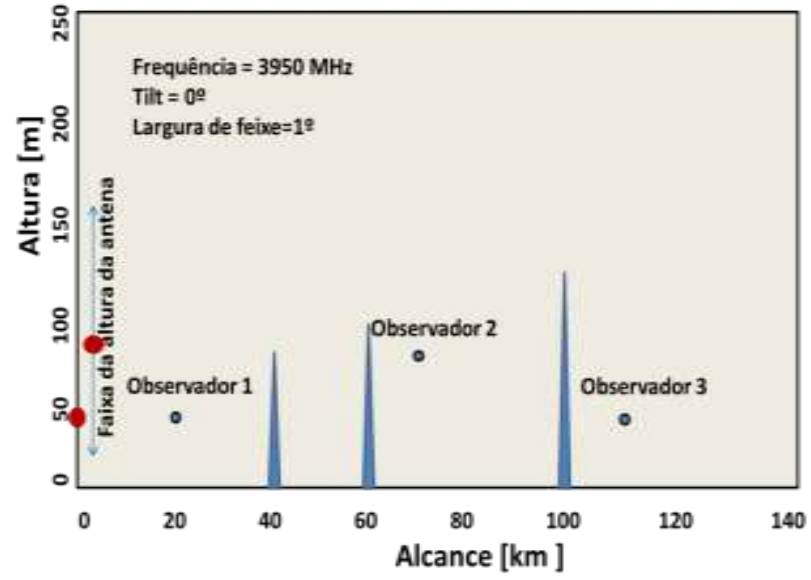

3.b) Configuração com obstáculos.

Fig. 3 Configurações do terreno com e sem obstáculos gume de faca 
Para analisar a influência do perfil de refratividade na propagação na troposfera, o módulo de propagação do EP-AG foi executado, utilizando as configurações de terreno da Figura 3 com os dois perfis de refratividade da Figura 4. O perfil de refratividade padrão mostrado na Figura 4.a é obtido pela equação (13)

$n(h)=1+a_{0} h$ equação (13)

com $\mathrm{a}_{0}=0,118 \mathrm{~m}^{-1}$ para qualquer altura. O perfil de refratividade com duto de superfície mostrado na Figura 4.b é obtido pela equação (14),

$$
n(h)=n_{0} \cdot\left\{\begin{array}{lr}
1+a_{0} h & h \leq H_{1} \\
1+a_{0} H_{1}+b_{0} h & H_{1}<h \leq H_{2} \\
1+a_{0} H_{1}+b_{0} H_{2}-a_{0} h & h>H_{2}
\end{array}\right.
$$

onde $\mathrm{h}$ representa a altura, $n_{0}$ a refratividade na superfície da Terra e os coeficientes $a_{0}$ e $b_{0}$ as inclinações dos gradientes de refratividade até $H_{1}$ e entre $H_{1}$ e $H_{2}$, respectivamente [4].

Para cada uma das configurações de terreno da Figura 3, o EP-AG foi executado para os dois perfis de refratividade da Figura 4. O valor do campo elétrico para todos os pontos de observação pode ser observado nos contornos apresentados na seção de resultados do modelo.

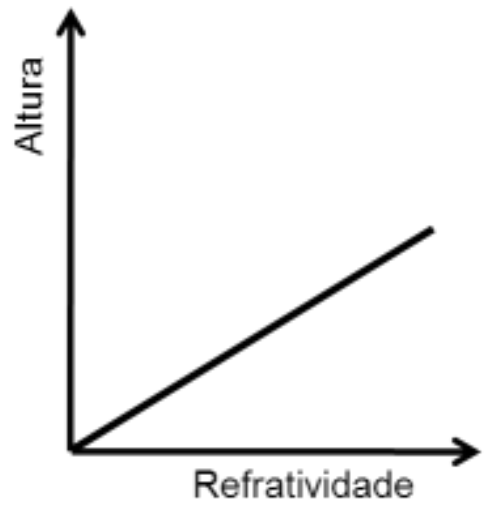

4.a) Perfil de Refratividade Padrão.

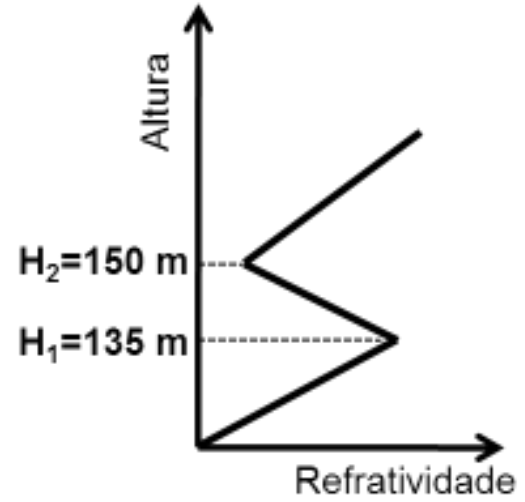

4b) Perfil de Refratividade com duto.

Fig. 4 Perfis de refratividade.

Para a otimização por Algoritmo Genético, foram utilizadas a codificação binária, seleção pelo método da roleta, reprodução por cruzamento, mutação e elitismo. Também foi utilizado um mecanismo de validação para garantir que todos os indivíduos atendam às restrições dos espaços 
de busca. O cálculo da aptidão foi realizado a partir da equação (12), considerando uma combinação dos parâmetros altura da antena e frequência de operação.

$\mathrm{Na}$ pesquisa de redução do custo computacional, os valores ótimos dos parâmetros foram determinados, utilizando a força bruta e a otimização por AG. Sendo que em ambos os procedimentos foram utilizadas a configuração de terreno da Figura 3.b e perfil de refratividade da Figura 4b. Para a frequência foram utilizadas, como espaço de busca, a faixa de 1000 a 4000 $\mathrm{MHz}$ e, para a altura da antena transmissora, a faixa de 30 a $150 \mathrm{~m}$. O ponto de observação escolhido para determinação do valor do campo elétrico como métrica da aptidão foi o observador 2. Este ponto foi escolhido por estar na metade do alcance máximo e entre dois obstáculos.

\section{RESULTADOS DO MODELO DE PROPAGAÇÃO}

O contorno do campo elétrico para a configuração da Figura 2 com tilt de $0^{\circ}$ pode ser visto na Figura 5, e para a mesma configuração, porém com tilt de $0,5^{\circ}$, pode ser visto na Figura 6.

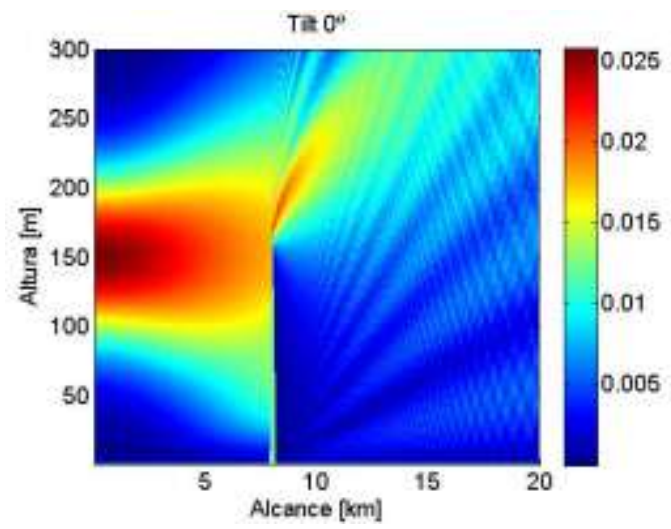

Fig. 5 Contorno do campo para tilt de $0^{\circ}$.

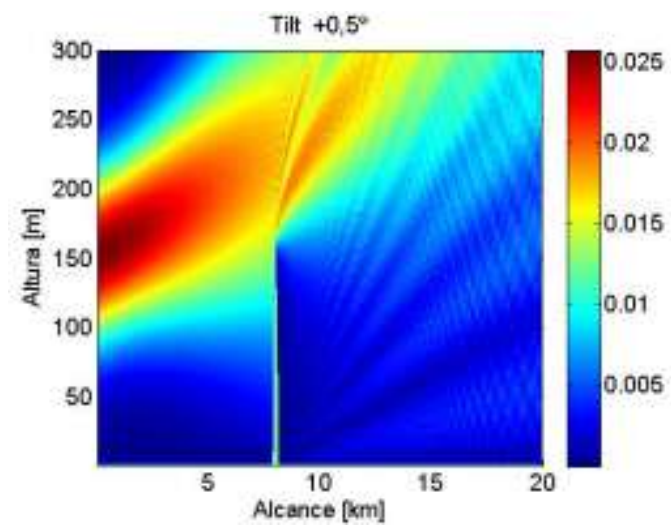

Fig. 6 Contorno do campo para tilt de $0,5^{\circ}$.

Analisando a Figuras 5, observa-se que para um observador antes do obstáculo a $4 \mathrm{~km}$ da antena, o campo é intenso na altura da antena, e diminui gradativamente à medida que ele se afasta para cima ou para baixo desta altura. Este fato é ratificado pelo perfil vertical do campo da Figura 7. Para o observador na posição do obstáculo, o perfil vertical do campo é mostrado na Figura 8. Observa-se que o campo é nulo até a altura do obstáculo. Esta é uma condição inerente ao algoritmo utilizado. Logo acima do obstáculo, o campo assume o valor máximo e vai decrescendo de forma oscilatória, sendo que a oscilação diminui a amplitude a partir dos $220 \mathrm{~m}$.

Na Figura 6, observa-se nitidamente que o feixe foi inclinado para cima, inclusive tem-se a impressão de que o ângulo tilt é bem maior que $0,5^{\circ}$. Esta falsa impressão ocorre porque a altura 
e o alcance estão em escalas diferentes. A escala da altura está em metros enquanto a do alcance em quilômetros.

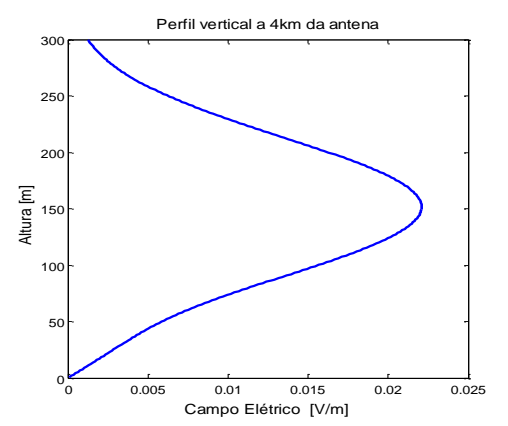

Fig. 7 Perfil vertical do campo antes do obstáculo para tilt de $0^{\circ}$.

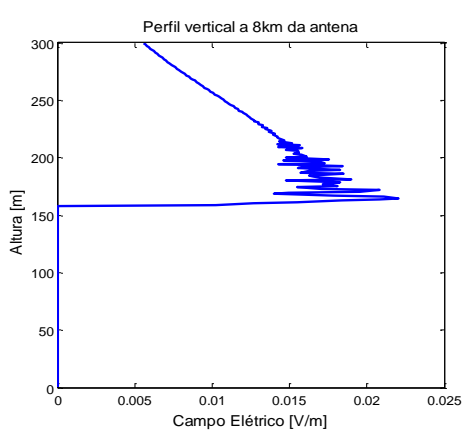

Fig. 8 Perfil vertical do campo na posição do obstáculo para tilt de $0^{\circ}$.

Os contornos do campo elétrico para análise da influência dos obstáculos e dos perfis de refratividade estão ilustrados nas Figuras de 9 a 12.

Comparando a Figura 10 com a Figura 9, observa-se claramente o efeito do duto, confinando a onda abaixo de 150 m, forçando-a a subir e descer várias vezes e alcançar grandes distâncias.

A Tabela 1 mostra o valor do campo elétrico nos três observadores destacados para todas as circunstâncias das Figuras 9 a 12.

No observador 1, o campo não altera o valor com a presença dos obstáculos, sendo de $-19,34$ $\mathrm{dBV} / \mathrm{m}$ para o gradiente de refratividade padrão e $-24,49 \mathrm{dBV} / \mathrm{m}$ para o duto de superfície. Isso ocorre porque o observador 1 está antes de todos os obstáculos.

No observador 2, a presença dos obstáculos diminui a intensidade do campo em $34 \mathrm{~dB}$ com o gradiente de refratividade padrão e $26 \mathrm{~dB}$ com o duto de superfície. Apesar dos obstáculos 1 e 2 estarem entre a antena transmissora e o observador 2 , percebe-se, pela figura 10 , que o duto contorna estes obstáculos e, consequentemente, eleva a intensidade do campo em $8 \mathrm{~dB}$ comparada com o gradiente de refratividade padrão.

No observador 3, a presença dos obstáculos diminui a intensidade do campo em 46,67 dB com a refratividade padrão e 50,29 dB com o duto de superfície. O duto de superfície não aumentou a intensidade do campo no observador 3 , uma vez que o mesmo está posicionado atrás do $3^{\circ}$ obstáculo, e este obstrui o duto. 


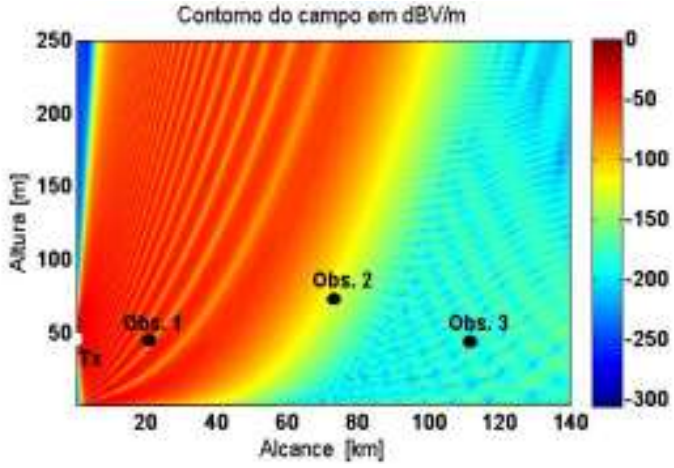

Fig. 9 Contorno do campo para terreno sem obstáculos e Refratividade padrão.

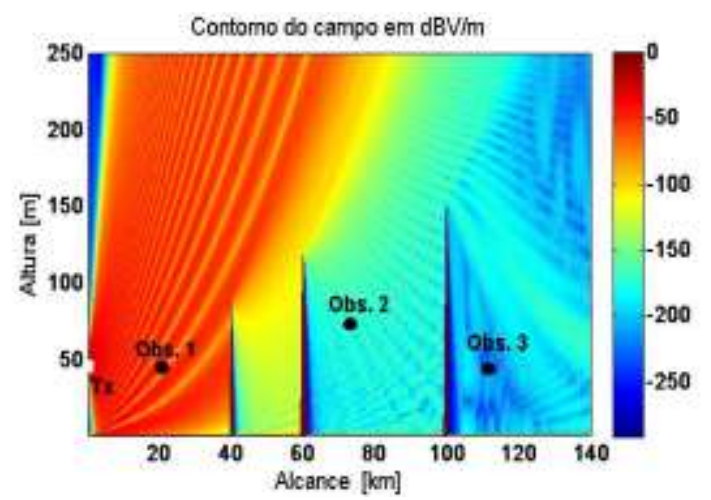

Fig. 11 Contorno do campo para terreno com obstáculos e Refratividade padrão.

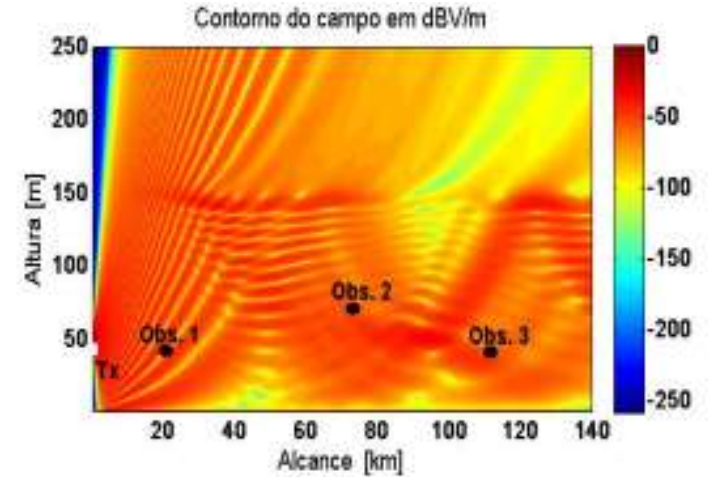

Fig. 10 Contorno do campo para terreno sem obstáculos e Refratividade com duto.

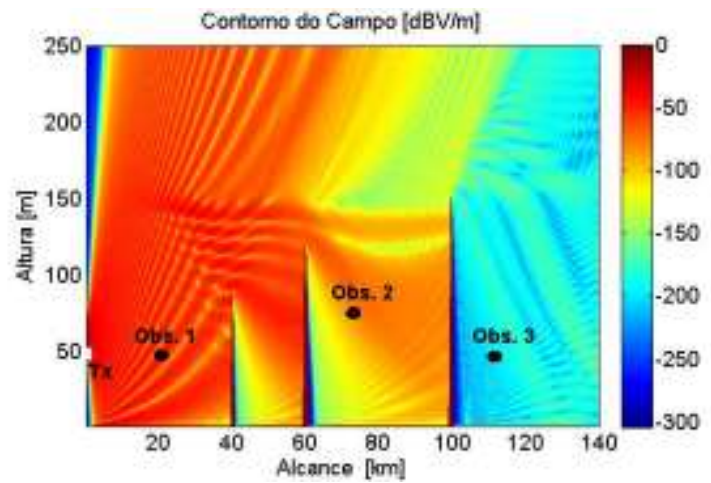

Fig. 12 Contorno do campo para terreno com obstáculos e Refratividade com duto.

Tabela 1 - Campo Elétrico [dBV/m] nos Observadores Destacados nas Figuras de 9 a 12.

\begin{tabular}{|c|c|c|c|c|}
\hline $\begin{array}{c}\text { Perfil de } \\
\text { Refratividade }\end{array}$ & $\begin{array}{c}\text { Presença de } \\
\text { Obstáculos }\end{array}$ & Observador 1 & Observador 2 & Observador 3 \\
\hline Padrão & Não & $-19,34$ & $-26,07$ & $-27,81$ \\
\hline Padrão & Sim & $-19,34$ & $-60,41$ & $-74,48$ \\
\hline Duto & Não & $-24,49$ & $-23,28$ & $-25,55$ \\
\hline Duto & Sim & $-24,49$ & $-49,88$ & $-75,84$ \\
\hline
\end{tabular}

\section{RESULTADOS DO MECANISMO DE OTIMIZAÇÃO}

Os tempos de processamento na busca de valores ótimos dos parâmetros altura da antena e frequência estão apresentados na tabela 2 e 3 para força bruta e otimização com AG, respectivamente. 
Tabela 2 - Processamento Pela Força Bruta.

\begin{tabular}{|c|c|c|c|c|}
\hline \multirow{2}{*}{$\begin{array}{c}\text { Número De } \\
\text { Amostras }\end{array}$} & \multirow{2}{*}{$\begin{array}{c}\text { Tempo de } \\
\text { processamento } \\
\text { [minutos] }\end{array}$} & \multirow{2}{*}{$\begin{array}{l}\text { Melhor } \\
\text { Solução } \\
{[\mathrm{dBV} / \mathrm{m}]}\end{array}$} & \multicolumn{2}{|c|}{ Valores Ótimos } \\
\hline & & & $h_{t x}[m]$ & $\mathrm{f}[\mathrm{MHz}]$ \\
\hline 10 & 16 & $-41,72$ & 126 & 2500 \\
\hline 20 & 62 & $-39,87$ & 132 & 3850 \\
\hline 40 & 303 & -39.74 & 132 & 3775 \\
\hline 80 & 901 & $-39,31$ & 132 & 3812 \\
\hline
\end{tabular}

Na tabela 4, é apresentada a redução do tempo de processamento com a utilização do AG e o erro dos resultados em relação ao resultado da força bruta com 80 amostras. Analisando os dados desta tabela, percebe-se que, apesar do tempo de processamento com AG ser bem menor, a precisão apresentada é praticamente a mesma alcançada com a força bruta.

Tabela 3 - Processamento com AG.

\begin{tabular}{|c|c|c|c|c|}
\hline \multirow{2}{*}{$\begin{array}{c}\text { Número De } \\
\text { Amostras }\end{array}$} & $\begin{array}{c}\text { Tempo de } \\
\text { processamento } \\
\text { [minutos] }\end{array}$ & $\begin{array}{c}\text { Melhor } \\
\text { Solução } \\
{[\mathrm{dBV} / \mathrm{m}]}\end{array}$ & \multicolumn{2}{|c|}{ Valores Ótimos } \\
\cline { 4 - 5 } & 16 & $-41,71$ & 126 & 1479 \\
\hline 10 & 30 & $-41,53$ & 121 & 2484 \\
\hline 20 & 63 & $-40,41$ & 134 & 3478 \\
\hline 40 & 121 & $-39,22$ & 132 & 3808 \\
\hline 80 & & &
\end{tabular}

Constatou-se que quanto maior o número de amostras, menor é o tempo de processamento do $A G$ em relação à Força Bruta. A razão disso é que o $A G$ determina o campo elétrico apenas para algumas combinações de parâmetros selecionadas e o número dessas combinações cresce de forma linear com o número de amostras, enquanto na força bruta o campo é determinado para todas as combinações possíveis do espaço de busca, e esse número cresce de forma exponencial com o número de amostras. 
Tabela 4 - Redução do Tempo de Processamento e Precisão.

\begin{tabular}{|c|c|c|c|}
\hline \multirow{2}{*}{$\begin{array}{c}\text { Número de } \\
\text { Amostras }\end{array}$} & \multirow{2}{*}{$\begin{array}{c}\text { Redução do } \\
\text { tempo de } \\
\text { Processamento }\end{array}$} & \multicolumn{2}{|c|}{ Erro em relação a FB com 80 amostras } \\
\cline { 3 - 4 } & $0 \%$ & $2,31 \%$ & Algoritmo Genético \\
\hline 10 & $43 \%$ & $1,91 \%$ & $1,54 \%$ \\
\hline 20 & $70 \%$ & $0,37 \%$ & $0,37 \%$ \\
\hline 40 & $85 \%$ & $0,00 \%$ & $0,00 \%$ \\
\hline 80 & & & $0,00 \%$ \\
\hline
\end{tabular}

Na Figura 13, pode ser vista a curva de desempenho do AG para 20 amostras. Essa curva é constituída dos valores máximo e médio da aptidão de cada geração. Percebe-se que a melhor solução nunca diminui devido ao elitismo. Percebe-se também que o valor médio cresce tendendo ao valor da melhor solução, que neste caso, ocorreu em torno da 13a geração, indicando que o algoritmo convergiu. A tabela 4 mostra a redução do tempo de processamento e o erro em relação a Força Bruta com 80 amostras.

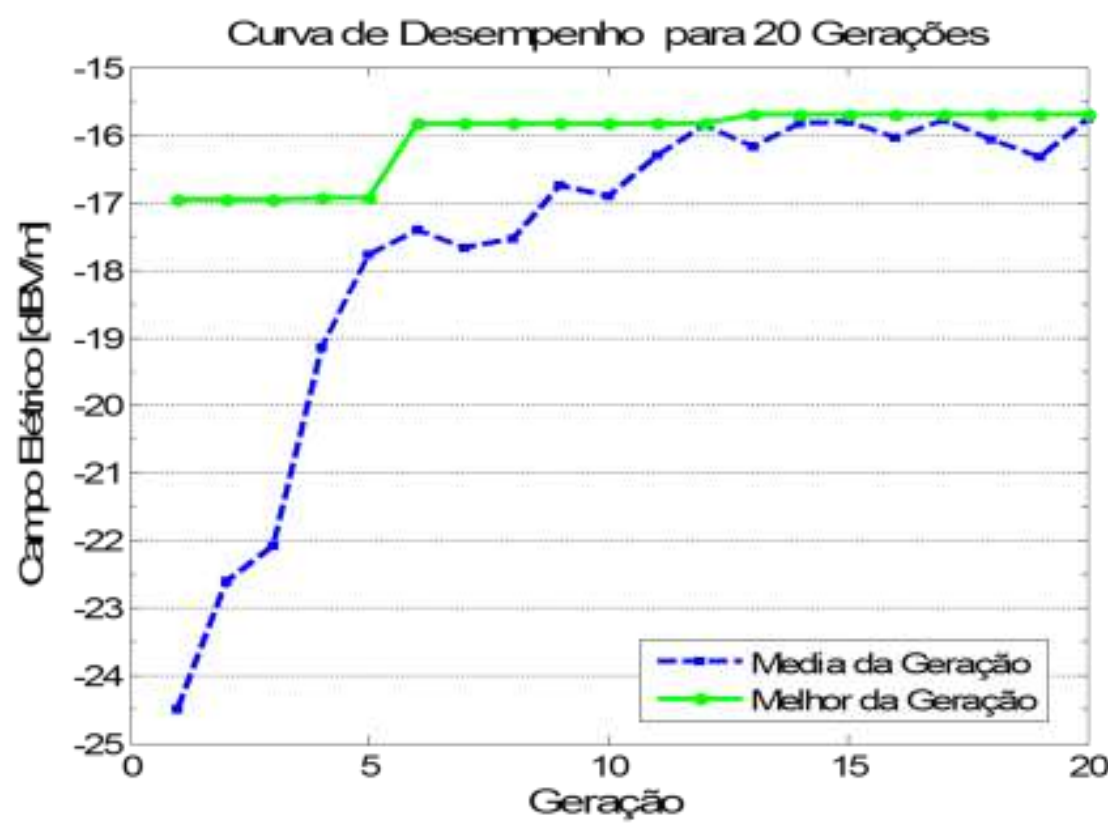

Fig.13 Curva de Desempenho para 20 Gerações e 20 amostras.

\section{CONCLUSÃO}

Este artigo apresentou um estudo de otimização estocástica, que utiliza algoritmos genéticos dos parâmetros altura da antena transmissora e frequência de operação em um modelo de 
propagação que utiliza equações parabólicas com o divisor de passos de Fourier. Os resultados obtidos demonstram que a utilização de algoritmos genéticos reduz significativamente o tempo de processamento na busca de valores ótimos dos parâmetros pesquisados, sem reduzir a precisão, o que implica na redução do custo computacional.

O próximo passo é empregar o AG para otimizar os parâmetros altura da antena e frequência em um modelo de propagação 3D com equações parabólicas. Tal modelo possui maior espaço de busca, o que ocasiona considerável impacto no tempo de processamento computacional. Portanto, a redução do custo computacional em tal modelo torna-se imperativo. A técnica de otimização empregada neste artigo poderá ser aplicada em outros modelos de propagação em suas diversas configurações.

\section{AGRADECIMENTOS}

Agradeço a CAPES pelo incentivo através do programa PIQDTec, bem como a FAPEMAT pelo auxilio financeiro na execução deste trabalho.

\section{REFERÊNCIAS BIBLIOGRÁFICAS}

1. Apaydin, G. and L. Sevgi, The Split-Step-Fourier and Finite-Element Based Parabolic-Equation Propagation Prediction Tools: Canonical Tests, Systematic Comparisons, and Calibration, in IEEE Transactions on Antennas and Propagation Magazine number, vol. 52, No 3, 2010. p. 66-79.

2. Gokhan, A., et al., Two-way Split-Step Fourier and Finite Element based Parabolic Equation Propagation Tools: Comparisons and Calibration, in Antennas and Propagation Society International Symposium vol. 49, IEEE 2010.

3. Hardin, R.H. and F.D. Tappert, Applications of the split-step Fourier method to the numerical solution of nonlinear and variable coefficient wave equations, , in SIAM , vol. 15, 1973. p. 423,1973.

4. Sevgi, L.e.a., A MATLAB-Based Two-Dimensional Parabolic Equation Radiowave Propagation Package, in IEEE Antennas and Propagation Magazine, vol. 47, No 4, 2005.

5. Ozgun, O., Recursive Two-Way Parabolic Equation Approach for Modeling Terrain Effects in Tropospheric Propagation in IEEE Transactions on Antennas and Propagation, vol. 57, No 9, 2009.

6. Ozgum, O., et al., Two-way Fourier Split Step Algorithm over Variable Terrain with Norrow and Wide angle Propagation, in Antennas and Propagation Society International Symposium number 50, IEEE 2010.

7. Barrios, A.E., A Terrain Parabolic Equation Model for Propagation in the Troposphere, in IEEE Transactions on Antennas and Propagation, vol. 42, No 1, 1994.

8. Ozgum, O., S.G. Tanyer, and C.B. Erol, An Examination of The Fourier Split-Step Method of Representing Electromagnetic Propagation in The Troposphere, in International Geoscience and Remote Sensing Symposium number 51, IEEE2002: Toronto - Canada. 
9. Lopes, H.S. and R.H.C. Takahashi, Computação Evolucionária em Problemas de Engenharia. 1a ed. 2011, Curitiba, PR: Omnipax Editora Ltda. 385 pages.

10. Ávila, S.L., Algoritmos Genéticos Aplicados na Otimização de Antenas Refletoras, in Dissertação UFSC. 2002: Florianópolis -SC.

11. Levy, M., Parabolic equation methods for electromagnetic wave propagation. IEE ELETROMAGNETIC WAVE SERIES 45 2000, London: The Institution of Eletrical Engineers.

12. Nascimento, V.E.d., Método FD-BPM Semivectorial de Ângulo Largo para a Análise de Estruturas Tridimensionais Utilizando a Técnica ADI, in Dissertação de Mestrado - USP. 2002: São Carlos -SP.

13. Goldberg, D.E., Genetic Algorittms in Search, Optimzation end MachineLearning. 1989: Addsun Wesley.

14. Bobel, E. and H.S. Lopes, Inteligência computacional aplicada à logística de equipes de manutenção de redes de distribuição de energia elétrica, in Espaço e Energia número 79, 2007. 